\title{
Acta
Biochimica
Polonica
}

Vol. 49 No. 1/2002

$99-107$

QUARTERLY

\section{The influence of intracellular idarubicin and daunorubicin levels on drug cytotoxicity in childhood acute leukemia ${ }^{\star \star}$}

\author{
Jan Styczyński ${ }^{1, \bowtie}$, Mariusz Wysocki ${ }^{1}$, Robert Dębski ${ }^{1}$, Andrzej Kurylak ${ }^{1}$, \\ Walentyna Balwierz ${ }^{2}$, Roma Rokicka-Milewska ${ }^{3}$, Michał Matysiak ${ }^{4}$, Anna Balcerska ${ }^{5}$, \\ Jerzy Kowalczyk $^{6}$, Jacek Wachowiak ${ }^{7}$, Danuta Sońta-Jakimczyk ${ }^{8}$ and Alicja Chybicka ${ }^{9}$ \\ ${ }^{1}$ Department of Pediatric Hematology and Oncology, Medical University, Bydgoszcz, Poland; \\ ${ }^{2}$ Department of Pediatric Hematology, Collegium Medicum Jagiellonian University, Kraków, \\ Poland; ${ }^{3}$ Department of Pediatric Hematology and Oncology, ${ }^{4}$ Department of Pediatric \\ Pulmonology, Alergology and Hematology, Medical University, Warszawa, Poland; ${ }^{5}$ Department \\ of Pediatric Hematology, Oncology and Endocrinology, Medical University, Gdańsk, Poland; \\ ${ }^{6}$ Department of Pediatric Hematology and Oncology, Medical University, Lublin, Poland; \\ ${ }^{7}$ Department of Pediatric Hematology and Oncology, Medical University, Poznań, Poland; \\ ${ }^{8}$ Department of Pediatric Hematology and Chemotherapy, Silesian Medical University, Zabrze, \\ Poland; ${ }^{9}$ Department of Pediatric Hematology and Oncology, Medical University, Wrocław, \\ Poland
}

Received: 10 September, 2001; revised: 03 January, 2002; accepted: 21 February, 2002

Key words: anthracyclines, acute lymphoblastic leukemia, acute myeloblastic leukemia, drug resistance, P-glycoprotein

\begin{abstract}
Uptake and efflux of two anthracyclines, idarubicin (IDA) and daunorubicin (DNR), was studied in childhood acute leukemia samples. A comparison of IDA and DNR transport phenomena in relation to drug cytotoxicity and expression of P-glycoprotein (PGP) was made. Intracellular content of IDA/DNR was determined by flow cytometry using the fluorescent properties of the drugs. In vitro drug cytotoxicity was
\end{abstract}

\footnotetext{
${ }^{\star}$ Presented at the 8th International Symposium on Molecular Aspects of Chemotherapy, September, 2001, Gdańsk, Poland.

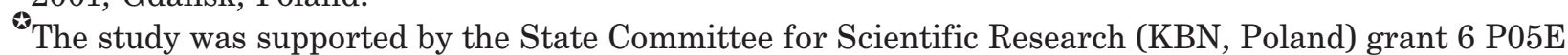
08221 and performed in the Laboratory of Clinical and Experimental Oncology, Department of Pediatric Hematology and Oncology, Medical University, Bydgoszcz, Poland.

${ }^{\otimes}$ Address for correspondence: Jan Styczyński, W. Gersona 17-8, 85-305 Bydgoszcz, Poland; fax: (48 52) 585 4867; e-mail: jan_styczynski@kki.net.pl
}

Abbreviations: ALL, acute lymphoblastic leukemia; iALL, initial ALL; rALL, relapsed ALL; AML, acute myeloblastic leukemia; BM, bone marrow; DNR, daunorubicin; FSC, forward scatter; IDA, idarubicin; PB, peripheral blood; PBS, phosphate-buffered saline; PGP, P-glycoprotein; RR, relative resistance; SSC, sideward scatter. 
measured by the 3-[4,5-dimethylthiazol-2-yl]-2,5-diphenyl tetrazolium bromide (MTT) assay. PGP expression was analysed by flow cytometry. The uptake and efflux rates were non-significantly higher for IDA than DNR. There were no differences between three types of leukemia with respect to drug content during accumulation and retention. After correction for the cell volume, intracellular concentration of both drugs in each moment of uptake and efflux was significantly lower in relapsed ALL and AML samples in comparison with initial ALL cells. Efflux, but not uptake, of both drugs was inversely correlated with PGP expression and IDA, but not DNR, cytotoxicity. The cytotoxicity was correlated with drug accumulation for both drugs and with drug retention for IDA. In conclusion, it seems that (1) intracellular content was related to the lipophilic properties of the drugs rather than to the type of leukemia, (2) decreased intracellular concentration of both drugs might have an impact on compromised therapy results in AML and relapsed ALL children, (3) IDA presents higher cytotoxicity, which possibly might be decreased by the presence of PGP. These results might have a practical impact on the rational design of new chemotherapy protocols.

Leukemia is the most common childhood malignancy. Acute lymphoblastic leukemia (ALL) is the predominant form (85\%) and acute myeloblastic leukemia (AML) is a relatively uncommon disease (10-15\%). With current protocols, $70-80 \%$ of children with ALL, and only $30-50 \%$ with AML can be cured [1]. Drug resistance is related to relapse of leukemia, but the underlying mechanisms are still poorly understood. Anthracyclines (Fig. 1) are

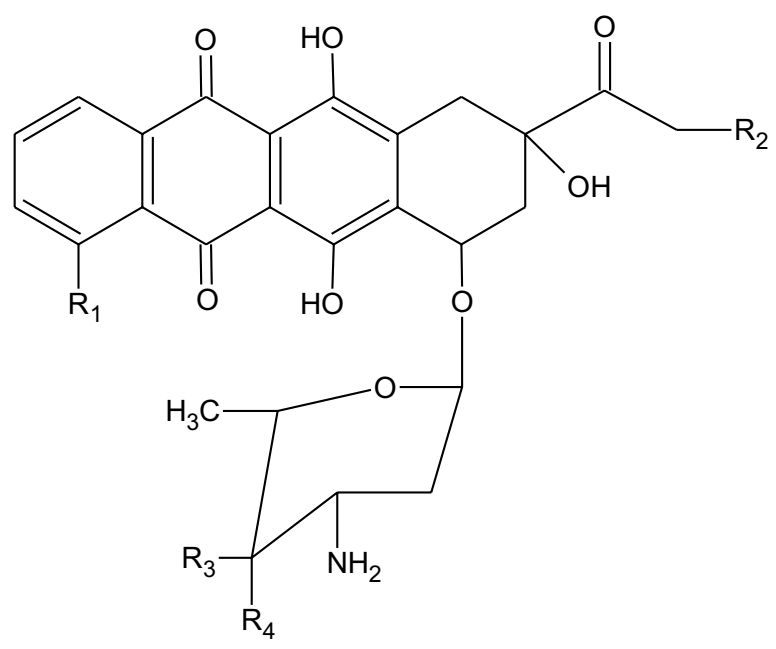

\begin{tabular}{|l|l|l|l|l|}
\hline & $\mathrm{R}_{1}$ & $\mathrm{R}_{2}$ & $\mathrm{R}_{3}$ & $\mathrm{R}_{4}$ \\
\hline daunorubicin & $\mathrm{OCH}_{3}$ & $\mathrm{H}$ & $\mathrm{H}$ & $\mathrm{OH}$ \\
\hline idarubicin & $\mathrm{OCH}_{3}$ & $\mathrm{H}$ & $\mathrm{H}$ & $\mathrm{OH}$ \\
\hline doxorubicin & $\mathrm{H}$ & $\mathrm{OH}$ & $\mathrm{H}$ & $\mathrm{OH}$ \\
\hline epirubicin & $\mathrm{OCH}_{3}$ & $\mathrm{OH}$ & $\mathrm{OH}$ & $\mathrm{H}$ \\
\hline
\end{tabular}

Figure 1. Chemical structure of anthracyclines. among the drugs used in both types of acute leukemia. Idarubicin (IDA) is a relatively new antitumor anthracycline drug and is thought to be more active and less cardiotoxic than daunorubicin (DNR) [2]. Resistance to anthracyclines may compromise therapy results. The resistance may be caused by reduced intracellular drug levels due to its sequestration by multidrug resistance proteins or detoxification by glutathione and its $S$-transferases, a reduced amount of active intracellular drug target, such as topoisomerase II, and resistance to apoptosis or its protein disregulation [3]. Anthracyclines enter the cell by passive diffusion, most likely in an unchanged form [4, 5]. So far most information about anthracycline resistance was obtained from in vitro studies on tumor cell lines. There is only one study on childhood acute leukemia, reporting a relationship between intracellular DNR concentration, expression of resistance proteins and DNR cytotoxicity in ALL [6]. It shows that intracellular DNR concentration and the expression of Lung-Resistance-Protein (MVP/LRP), but not P-glycoprotein (PGP), may contribute to DNR resistance in childhood ALL. So far there are no reports available showing whether intracellular IDA content is related to cellular drug resistance and multi-drug resistance proteins expression in childhood leukemia. Moreover, it is unknown what differences in intracellular anthracycline levels occur between ALL and 
AML, and if they might contribute to differences in drug resistance.

The scope of this study is an analysis of uptake and efflux rates as well as intracellular IDA content and concentration in childhood acute leukemia. Another objective is a comparison of IDA and DNR transport phenomena in relation to cytotoxicity and expression of PGP.

\section{MATERIALS AND METHODS}

Leukemic cell samples. Samples of leukemic cells of 61 children aged $0.5-18$ yrs (median $8 \mathrm{yrs}$ ), including 34 with ALL de novo (iALL, group A), 13 relapsed ALL (rALL, group B) and 14 de novo AML (iAML, group C), were analysed. Only fresh peripheral blood (PB) or bone marrow (BM) samples taken at the moment of diagnosis or relapse were analysed. Cells were isolated by centrifugation on Ficoll gradient at $480 \times \mathbf{g}$ for $20 \mathrm{~min}$ at room temperature, washed twice and resuspended in RPMI-1640 (Sigma, St. Louis, MO, U.S.A.) with $20 \%$ fetal calf serum (FCS, Gibco BRL, Life Technologies, Germany), $2 \mathrm{mM}$ L-glutamine, $5 \mu \mathrm{g} / \mathrm{ml}$ insulin, $5 \mu \mathrm{g} / \mathrm{ml}$ transferrin, $5 \mathrm{ng} / \mathrm{ml}$ sodium selenite (all from Sigma) and antibiotics. All samples presented at least 95\% cell viability (determined morphologically by trypan blue exclusion assay) and contained at least $80 \%$ of leukemic cells, as calculated on cytospin slides stained with the May-Grunwald-Giemsa method. The concentration of leukemic cells was adjusted to $2 \times 10^{6} / \mathrm{ml}$.

Drugs. Daunorubicin (Cerubidine; Rhone Poulenc Rorer, Amstelveen, The Netherlands) and idarubicin (Zavedos; Pharmacia Farmitalia Oncology, Italy) were used for the study.

Drug accumulation and retention. Leukemic cells at a concentration of $0.5 \times 10^{6}$ cells $/ \mathrm{ml}$ were incubated with $10 \mu \mathrm{g} / \mathrm{ml}$ of respective drug in humidified air containing $5 \%$ $\mathrm{CO}_{2}$ at $37^{\circ} \mathrm{C}$. Samples of cells were analysed in $1 \mathrm{~h}$ intervals. Accumulation of DNR/IDA was determined at certain time points during continuous exposure to DNR/IDA. To study the retention, cells were first loaded with $10 \mu \mathrm{g} / \mathrm{ml} \mathrm{DNR} / \mathrm{IDA}$ for $1 \mathrm{~h}$ at $37^{\circ} \mathrm{C}$. In order to start the efflux, cells were washed in ice-cold phosphate-buffered saline (PBS) and resuspended in a pre-warmed drug-free medium. The retained amount of DNR/IDA was determined at several time points, including the start of efflux $(t=0)$. Each aliquot $(100 \mu \mathrm{l})$ was washed immediately in ice-cold PBS and then intracellular DNR/IDA fluorescence (FL) was measured by flow cytometry (Coulter EPICS XL3, Miami, FL, U.S.A.). The content of intracellular IDA/DNR was determined by flow cytometry using the fluorescent properties of the drugs. DNR/IDA was excitated by an argon laser at $488 \mathrm{~nm}$ and the fluorescence signal was collected through a 515-535 nm (FL1 height), 565-585 nm (FL2 height) and 610-630 nm (FL3 height) bandpass filter set. The strongest emission was observed for the FL2 filter for IDA and FL3 filter for DNR. The instrument settings for FL2 and FL3 heights, forward scatter (FSC) and sideward scatter (SSC) were kept constant between two runs of the flow cytometer. The data were analysed using the SYSTEM II version 3.0 software (Beckman Coulter, Miami, FL, U.S.A.). Viable leukemic cells were gated based on FSC and SSC characteristics. The percentage of gated cells corresponded well with the percentage of viable cells determined morphologically by trypan blue exclusion. The intracellular drug content was determined by the mean fluorescence intensity of gated leukemic cells, and was expressed in arbitrary units (AU) on a log-scale. The FSC of gated leukemic cells was used as a relative measure of cell volume $\left(\mathrm{FSC}^{3}\right)$ in this study. The intracellular drug concentration (cDNR/cIDA) was obtained by dividing the intracellular DNR/IDA content by the relative cell volume (AU/FSC ${ }^{3}$ ) [6]. The percentage of drug retention was calculated by dividing the drug-fluorescence retained in the cell after a period of efflux by the drug-fluorescence pres- 
ent at the start of efflux $(t=0) \times 100 \%$. The intracellular drug content, intracellular drug concentration or percentage of drug retention did not differ between bone marrow and peripheral blood samples. Therefore, those data were pooled for further analysis.

In vitro drug resistance assay. The cytotoxicity of the drugs was measured by the MTT assay. The assay conditions were essentially the same as described previously [7]. Briefly, $80 \mu \mathrm{l}$ of the cell suspension containing $2 \times 10^{6}$ vital cells $/ \mathrm{ml}$ was incubated with each drug concentration (drug was added as a solution in $20 \mu \mathrm{l}$ RPMI) in duplicate wells of a 96-well round-bottomed microtiter plate (Profilab, Warsaw, Poland). Six wells containing only cells in drug-free medium served as controls for cell survival, while six other wells containing only culture medium blanked the spectrophotometer. The outer wells of the plate were filled with RPMI-1640 only. Plates were then wrapped in cling film and incubated for 4 days ( $96 \mathrm{~h}$ ) at $37^{\circ} \mathrm{C}$ in humidified air containing $5 \% \mathrm{CO}_{2}$. After 4 days, $50 \mu \mathrm{g}$ (10 $\mu \mathrm{l}$ of a $5 \mathrm{mg} / \mathrm{ml} \mathrm{solution)}$ of 3-[4,5-dimethylthiazol-2-yl]-2,5-diphenyl tetrazolium bromide (MTT, Serva, Heidelberg, Germany) was added to each well (final concentration 0.45 $\mathrm{mg} / \mathrm{ml}$ ); plates were shaken and incubated for another $6 \mathrm{~h}$ at $37^{\circ} \mathrm{C}$. In such an exposure yellow MTT was reduced to purple formazan by viable, but not dead, cells. The formazan crystals were dissolved with $100 \mu \mathrm{l}$ of acidified (0.04 M HCl) 2-isopropanolol (Sigma, St. Louis, MO, U.S.A.) and the quantity of the reduced product was measured by an ELISA EL-312 microplate spectrophotometer at 570 nm (Asys Hitech GmbH, Eugendorf, Austria). The absorbance $\left(\mathrm{A}_{570}\right)$ at this wavelength is linearly related to the number of viable cells [8]. Cytospin slides from control wells, stained with May-Grunwald-Giemsa, were used to determine the percentage of blasts after 96-h incubation. Samples with more than $70 \%$ leukemic cells in the control wells without drug after 4 days of culture and with an $\mathrm{A}_{570}$ higher than 0.050 arbitrary units (adjusted for blank values) were considered suitable for evaluation. The leukemic cell survival was calculated by the equation: $\left(\mathrm{A}_{570}\right.$ drug well/mean $\mathrm{A}_{570}$ control wells) $\times 100 \%$. The $A_{570}$ values of both control and tested wells were adjusted by the $\mathrm{A}_{570}$ of blank wells. The $\mathrm{LC}_{50}$ value, expressing the concentration of the drug that was lethal to $50 \%$ of the cells, was used as a measure of the in vitro drug cytotoxicity in each sample.

PGP expression. PGP was analysed by flow cytometry. Mouse monoclonal antibodies anti-PGP-PE (cat. No. 340555, Becton Dickinson, San Jose, CA, U.S.A.), clone 15D3 transfected with the human multidrug resistance gene MDR1, were used. The amount of PE-labelling was detected by flow cytometry; red fluorescence was collected through a FL2 height bandpass filter set using log-mode amplification. Leukemic cells were gated based on FSC and SSC characteristics, and the fluorescence intensity of this population was expressed in arbitrary units on a log-scale. PGP expression was calculated as percentage of cells positive against isotype control of mouse anti-IgG1-PE. No difference between the fluorescence of bone marrow and peripheral blood samples was observed, therefore those data were pooled for further analysis.

Statistics. The non-parametric Wilcoxon matched-pairs signed ranks test was used to compare $\mathrm{LC}_{50}$ values for $\mathrm{PB}$ and $\mathrm{BM}$ samples. The Mann-Whitney U test was used to compare accumulation and retention rates, as well as drug resistance, between groups. Correlations between parameters were calculated with Spearman's rho coefficient. All tests were used for two-tailed testing at a level of significance of 0.05 .

\section{RESULTS}

The results (intracellular concentration, PGP expression, cytotoxicity) showed large interpatient variability. There was a big overlap of results inside each group of patients. 
The uptake and efflux rates were higher for IDA, however, the differences were not significant. There were no statistical differences within any of the three groups of patients with respect to drug content during accumulation
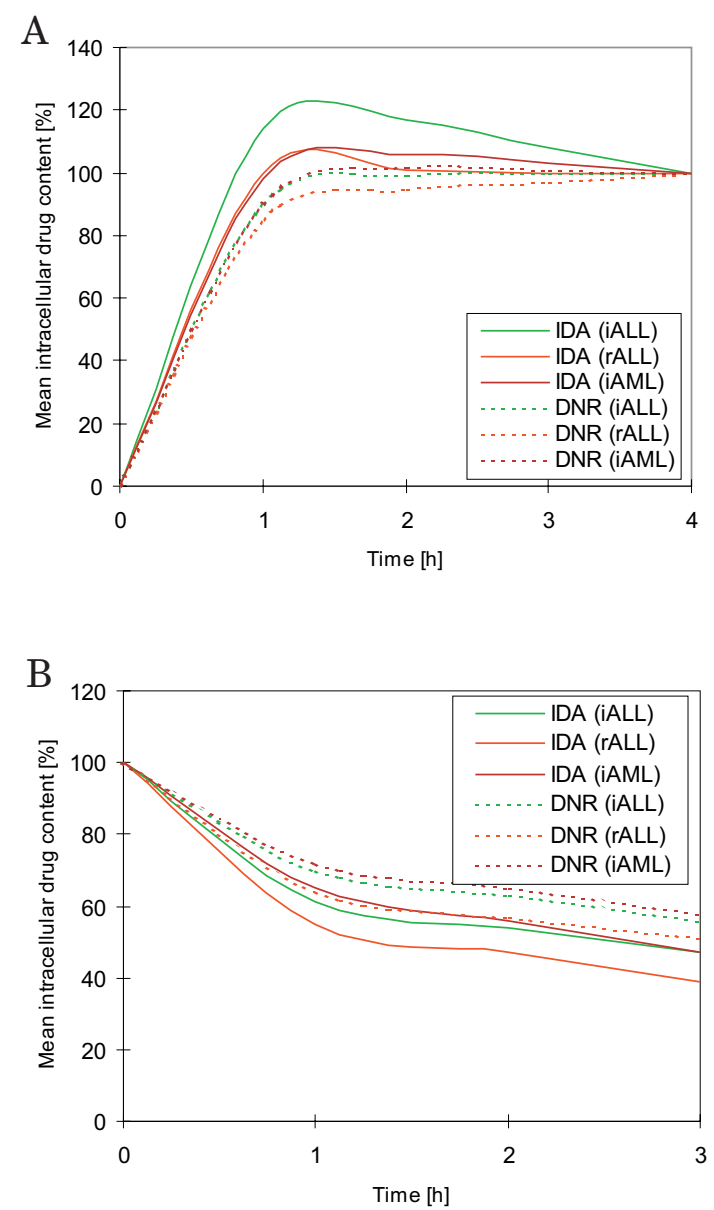

Figure 2. Rate of accumulation (A) and retention (B) of IDA and DNR in IALL, rALL and iAML cell samples.

Cells were incubated with each drug at of $10 \mu \mathrm{g} / \mathrm{ml}$. The intracellular drug content at each time point is given as the percentage of respective value at the end (accumulation phase) or at the beginning of the experiment (retention phase).

and retention (Fig. 2). It seems that the higher rate of IDA transport was related to the lipophilic properties of the drugs rather than to the type of leukemia.

However, when the cell volume was used to calculate the intracellular drug concentration based on its intracellular content, differences were found. Intracellular concentration of each of the two drugs at each time point of both uptake and efflux (Table 1, Fig. 3) was significantly lower in relapsed ALL (group B) and AML (group C) samples than in initial ALL cells (group A). Those differences were related to the cell volumes larger in groups B and $\mathrm{C}$ than A (Table 2).

There was no correlation between both drug accumulation and PGP expression at any time point during uptake. There was an inverse correlation between intracellular concentration of both drugs during efflux and PGP expression almost at each time point for all 61 patients (but not in any smaller group of patients with specific type of the disease) (Table 3$)$. The in vitro cytotoxicity expressed as $\mathrm{LC}_{50}$ was correlated with drug retention for IDA ( $p=0.012$ after $1 \mathrm{~h}$ and $\mathrm{p}=0.024$ after $2 \mathrm{~h}$ ) but not DNR. The correlation between $\mathrm{LC}_{50}$ and intracellular concentration during accumulation showed weak significance for both drugs $(\mathrm{p}<0.05)$. The $\mathrm{LC}_{50}$ values for DNR and IDA did not differ, whether in the whole group of patients (median $0.3594 \mu \mathrm{g} / \mathrm{ml}$ vs $0.3596 \mu \mathrm{g} / \mathrm{ml}$ ) or in any subgroup, however, for each drug differences were observed between the three types of leukemia (Table 4).

\section{DISCUSSION}

Anthracyclines are among the most commonly used anti-tumor drugs ever developed. The first identified anthracyclines, daunorubicin and doxorubicin, were isolated from pigment-producing Streptomyces species in the early 1960s [9]. The 4-demethoxy derivative of daunorubicin, idarubicin, is more effective than the parent compound in HL60 cell line [10]. Resistance to DNR has been shown to correlate with the prognosis of pediatric patients with ALL [11]. The present study tested whether decreased cellular uptake or increased cellular efflux of DNR and IDA may be responsible for cellular DNR/IDA resistance in childhood ALL and AML. The 
Table 1. Intracellular concentration of DNR and IDA during uptake and efflux

\begin{tabular}{|c|c|c|c|c|c|c|}
\hline \multirow[b]{2}{*}{ Time $[\mathrm{h}]$} & \multicolumn{3}{|c|}{ Intracellular DNR concentration } & \multicolumn{3}{|c|}{ Intracellular IDA concentration } \\
\hline & 1 & 2 & 3 & 1 & 2 & 3 \\
\hline \multicolumn{7}{|l|}{ Uptake } \\
\hline iALL & 19 & 19 & 19 & 22 & 21 & 20 \\
\hline rALL & $11^{*}$ & $11^{* *}$ & $12^{* *}$ & $12^{*}$ & $13^{*}$ & 14 \\
\hline iAML & $6^{* * *}$ & $6.4^{* * *}$ & $6^{* * *}$ & $8.7^{* * *}$ & $8^{* *}$ & $8.1^{* * *}$ \\
\hline \multicolumn{7}{|l|}{ Efflux } \\
\hline iALL & 12 & 8.3 & 6.5 & 14 & 9.1 & 5.7 \\
\hline rALL & $7.6^{* *}$ & $4.0^{* *}$ & $3.4^{* * *}$ & $9 *$ & $5.5^{* *}$ & $3^{* * *}$ \\
\hline iAML & $3.7^{* * *}$ & $2.7^{* * *}$ & $2.2^{* * *}$ & $5.6^{* * *}$ & $3.1^{* * *}$ & $2.6^{* * *}$ \\
\hline
\end{tabular}

The values of drug concentration are given as median concentration (AU/FSC ${ }^{3}$ ) $\times 10^{7}$. Significance: $P<0.05,{ }^{* *} P<0.01,{ }^{* * *} P<$ 0.001 in relation to iALL.

intracellular anthracycline levels may be lowered by efflux-mediating proteins, such as PGP, MRP and MVP/LRP [6, 12], but it is thought that the role of these proteins in childhood leukemia is limited [13]. Previously, it has been shown that the transport rate de- differences in the intracellular content of IDA/DNR between the types of leukemia, there were significant differences in drug concentration. Larger cells were more resistant to either anthracycline. When the intracellular DNR content was corrected for cell

Table 2. Median cell volume and relative resistance of PALL and iAML $v$ s iALL

\begin{tabular}{llll}
\hline Group & Cell volume $\left(\mathrm{FSC}^{3}\right)$ & $\mathrm{RR}$ & $\mathrm{p}$ \\
\hline iALL & $3.1 \times 10^{-7}$ & - & - \\
rALL & $4.4 \times 10^{-7}$ & $1.4^{*}$ & 0.006 \\
iAML & $11.0 \times 10^{-7}$ & $3.5^{* *}$ & $<0.001$ \\
\hline
\end{tabular}

$\mathrm{RR}$, relative resistance in comparison to iALL; (*) RR is the median $\mathrm{LC}_{50}$ for $\mathrm{rALL}$ divided by the median $\mathrm{LC}$ 50 for iALL; $\left(^{* *}\right) \mathrm{RR}$ is the median $\mathrm{LC}_{50}$ for iAML divided by the median $\mathrm{LC}_{50}$ for $\mathrm{iALL}$; $\mathrm{RR}>1$ indicates that for a given drug, rALL (iAML) is more resistant than iALL; p, Mann-Whitney U test.

pends on the hydrophobicity of the drug: more lipophilic anthracyclines such as IDA enter the cell faster compared with the less hydrophobic DNR [14]. We observed that in childhood leukemic cells, IDA is not only accumulated faster than DNR, but it is also faster sequestered out of the cell. This may have an impact on the higher IDA cytotoxicity. The accumulation rates for both drugs were inversely correlated with $\mathrm{LC}_{50}$ values, reflecting drug sensitivity. On the other hand, faster IDA efflux could be related to the cellular resistance to this drug. Another important observation of the study is that while there were no volume, the intracellular DNR/IDA concentration inversely correlated with DNR/IDA resistance, both when the accumulation and retention of the drug were studied. These data show that not the decreased intracellular content but rather the decreased intracellular concentration may be an important mechanism of resistance which plays a role in the cytotoxic effect of both anthracyclines in childhood leukemia. Those observations, together with earlier results [6], allowed us to draw more general conclusions. The highest concentration of each drug was in iALL, in correlation with the lowest drug resistance 
A

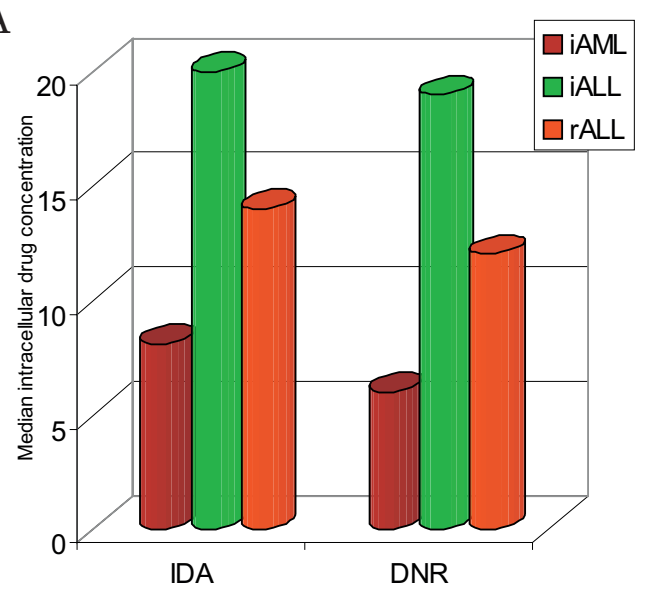

B

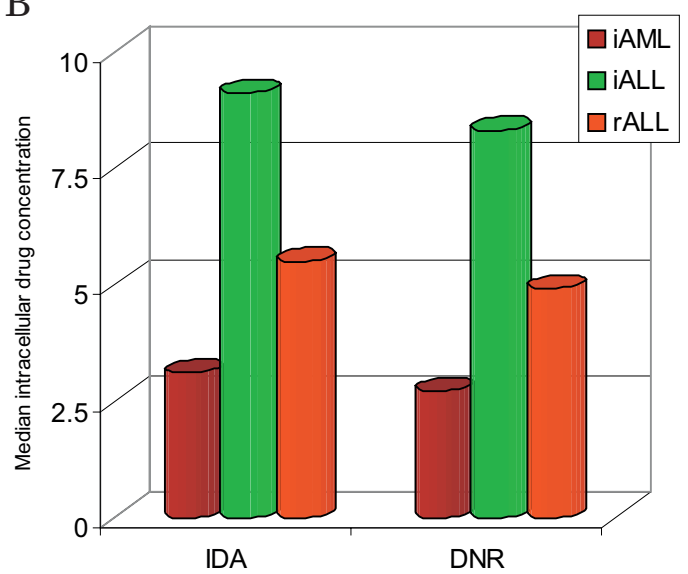

Figure 3. Intracellular concentration of IDA and DNR in iALL, rALL and iAML samples after $3 \mathrm{~h}$ of uptake (A) and efflux (B).

and the best clinical results of therapy. The intracellular DNR/IDA concentration was much lower in rALL and iAML, in correlation with higher drug resistance and much worse results of anti-leukemic therapy in the pediatric clinic. Finally, we observed that PGP expression may be one of the factors influencing intracellular concentration of drugs and cytotoxicity profile. PGP correlated with the intracellular DNR/IDA concentration during efflux. This is probably due to the physiological role of PGP, which helps to sequestrate drugs outside the cells during efflux but rather cannot influence the intracellular drug concentration during accumulation, since the drug uptake is more dependent on passive drug diffusion. It could also have a certain effect on IDA retention (as IDA was faster sequestrated out of the cells), however, this effect can have only minor clinical significance, since IDA caused very fast cell death. We frequently observed that after $3 \mathrm{~h}$ of accumulation most cells were killed by IDA at a concentration of $10 \mu \mathrm{g} / \mathrm{ml}$.

In conclusion, while the intracellular content of both anthracyclines was similar during uptake and efflux in all groups of patients, the intracellular concentration of the drugs was significantly decreased in AML and relapsed ALL children. This possibly indicates an important drug resistance mechanism in childhood acute leukemia. Retention of both drugs weakly correlated with PGP expression and IDA, but not DNR, cytotoxicity. These observations suggest higher IDA cytotoxicity, possibly decreased by the presence of PGP. These results might have a practical impact on the rational design of new chemotherapy protocols. On the other hand, anthracycline accumulation can possibly be decreased also due

Table 3. Correlation between intracellular drug concentration changes during efflux and PGP expression for all patients*

\begin{tabular}{lllll}
\hline \multirow{2}{*}{ Time points } & \multicolumn{2}{l}{ Intracellular DNR concentration } & \multicolumn{2}{l}{ Intracellular IDA concentration } \\
\cline { 2 - 5 } & rho & $\mathrm{p}$ & rho & $\mathrm{p}$ \\
\hline $1 \mathrm{~h}$ & -0.342 & 0.025 & -0.377 & 0.013 \\
$2 \mathrm{~h}$ & -0.388 & 0.012 & -0.369 & 0.018 \\
$3 \mathrm{~h}$ & -0.348 & 0.022 & -0.238 & 0.124 \\
\hline
\end{tabular}

*No correlations were found within any leukemia subgroup; rho, Spearman's coefficient of correlation; p, Mann-Whitney U test. 
Table 4. Cytotoxicity of idarubicin and daunorubicin

\begin{tabular}{lllllll}
\hline & \multicolumn{5}{c}{ IDA } & \multicolumn{2}{l}{ DNR } \\
\cline { 2 - 7 } & $\begin{array}{l}\text { Median } \mathrm{LC}_{50} \\
\mu \mathrm{g} / \mathrm{ml}\end{array}$ & $\mathrm{RR}$ & $\mathrm{p}$ & $\begin{array}{l}\text { Median } \mathrm{LC}_{50} \\
\mu \mathrm{g} / \mathrm{ml}\end{array}$ & $\mathrm{RR}$ & $\mathrm{p}$ \\
\cline { 2 - 5 } & 0.29 & - & - & 0.32 & - & - \\
rALL & 0.45 & $1.5^{*}$ & 0.008 & 0.38 & $1.2^{*}$ & 0.109 \\
iAML & 0.52 & $1.8^{* *}$ & 0.007 & 0.76 & $2.3^{* *}$ & 0.003 \\
\hline
\end{tabular}

$\mathrm{RR}$, relative resistance in comparison with iALL; ( $\left.{ }^{*}\right) \mathrm{RR}$ is the median $\mathrm{LC}_{50}$ for rALL divided by the median $\mathrm{LC}$ 50 for iALL; (**) $\mathrm{RR}$ is the median $\mathrm{LC}_{50}$ for iAML divided by the median $\mathrm{LC}_{50}$ for $\mathrm{iALL} ; \mathrm{RR}>1$ indicates that for a given drug, rALL (iAML) is more resistant than iALL; $p$, Mann-Whitney U test.

to the presence of other drug resistance proteins, such as Multidrug-Resistance Related Protein (MRP) or Lung-Resistance-Protein (MVP/LRP) [1].

Authors thank Małgorzata Kubicka, MSc., and Beata Kołodziej for technical assistance.

\section{R E F E R E N C E S}

1. Styczyński, J. \& Wysocki, M. (2000) Mechanisms of drug resistance in leukemias: Therapeutic problem. Adv. Clin. Exp. Med. 9, 153-161.

2. Hortobagyi, G.N. (1997) Anthracyclines in the treatment of cancer. Drugs 54 (Suppl. 4), 1-7.

3. McKenna, S.L. \& Padua, R.A. (1997) Multidrug resistance in leukemia. Br. J. Haematol. 96, 659-674.

4. Skovsgaard, T. \& Nissen, N.I. (1982) Membrane transport of anthracyclines. Pharmacol. Ther. 18, 293-311.

5. Siegfried, J.M., Burke, T.G. \& Tritton, T.R. (1985) Cellular transport of anthracyclines by passive diffusion. Biochem. Pharmacol. 34, 593-598.

6. Den Boer, M.L., Pieters, R., Kazemier, K.M., Janka-Schaub, G.E., Henze, G. \& Veerman, A.J.P. (1999) Relationship between the intacellular daunorubicin concentration, expression of major vault protein/lung resis- tance protein and resistance to anthracyclines in childhood acute lymphoblastic leukemia. Leukemia 13, 2023-2030.

7. Styczyński, J., Pieters, R., Huismans, D.R., Schuurhuis, G.J., Wysocki, M. \& Veerman, A.J.P. (2000) In vitro drug resistance profiles in adult versus childhood acute lymphoblastic leukaemia. Br. J. Haematol. 110, 813-818.

8. Kaspers, G.J.L., Veerman, A.J.P., Pieters, R., Van Zantwijk, C.H., Klumper, E., Hählen, K., De Waal, F.C. \& Van Wering, E.R. (1994) In vitro cytotoxicity of mitoxantrone, daunorubicin and doxorubicin in untreated childhood acute leukemia. Leukemia 8, 24-29.

9. Weiss, RB. (1992) The antracyclines: Will we ever find a better doxorubicin? Semin. Oncol. 19, 670-686.

10. Smeets, M.E.P., Raymakers, R.A.P., Vierwinden, G., Pennings, A.H.M., Boezman, J., Minderman, H. \& De Witte, T.M. (1999) Idarubicin DNA intercalation is reduced by MRP1 and not PGP. Leukemia 13, 13901398.

11. Pieters, R., Huismans, D.R., Loonen, A.H., Hählen, K., Van Der Does-Van Den Berg, A., Van Wering, E.R. \& Veerman, A.J.P. (1991) Relation of cellular drug resistance to long-term clinical outcome in childhood acute lymphoblastic leukaemia. Lancet 338, 399-403.

12.Loe, D.W., Deeley, R.G. \& Cole, S.P.C. (1996) Biology of the multidrug resistance mediated 
by the multidrug resistance-associated protein, MRP. Eur. J. Cancer 32A, 945-957.

13. Van den Heuvel-Eibrink, M.M., Sonneveld, P. \& Pieters, R. (2000) The prognostic significance of membrane transport-associated multidrug resistance (MDR) proteins in leukemia. Int. J. Clin. Pharmacol. Ther. 38, 94-110.
14. Berman, E. \& McBride, M. (1992) Comparative cellular pharmacology of daunorubicin and idarubicin in human multidrug-resistant leukemia cells. Blood 79, 3267-3273. 